\title{
X-ray analysis of protein crystals with thin-plate morphology
}

\author{
Olga Mayans and Matthias Wilmanns ${ }^{\star}$ \\ European Molecular Biology Laboratory (EMBL), EMBL Hamburg Outstation, c/o DESY, \\ Notkestrasse 85, D-22603 Hamburg, Germany.E-mail: wilmanns@embl-hamburg.de
}

\begin{abstract}
A case study on quasi two-dimensional crystals of the serine kinase domain of the giant muscle protein titin is presented. These crystals were obtained from several precipitating agents. However, under all conditions their growth in the third dimension was inhibited resulting in very thin plates, hardly visible under the microscope. Here a number of aspects that have been essential for highresolution data collection from these crystals are described: (i) improvement of the crystals by fine tuning their growth, namely by the addition of organic solvents, the application of macroseeding, and the reduction of vapour diffusion in the crystal drop vials using oil mixtures over the reservoir solutions; (ii) cryomounting techniques preventing mechanical stress; and (iii) optimizing the use of synchrotron radiation.
\end{abstract}

Keywords: protein crystals; plate morphology; titin kinase.

\section{Introduction}

Crystallographic studies of biological macromolecules have been facilitated by advances in phasing methods and the availability of an increasing number of synchrotron facilities for structural biology. With the increasing complexity of biological projects tackled by crystallographic methods, the process of sample preparation often becomes the ratelimiting step in the structural analysis. The preparatory process includes overexpression, purification and crystallization of the sample. Often crystals grow but do not appear to be feasible for X-ray analysis, and improving the quality of the crystals can be a difficult task. The search for better crystals often results in time-consuming repetitions of the preparatory cycle for different constructs for crystallization trials.

Here we present a case study where crystals of the serine kinase domain of the giant muscle protein titin, referred to as titin kinase (TK), were available for about four years but the structure solution was hampered because no X-ray data of sufficient quality could be collected. Although crystal plates appeared from various precipitating agents, they never acquired a measurable thickness. We describe and discuss a number of aspects in crystallization and mounting of the TK crystals which finally allowed us to collect a complete data set and to solve the crystal structure (Mayans et al., 1998). The availability of X-ray synchrotron radiation has been essential for crystal characterization and data collection.

\section{Crystallization of TK}

For all crystallization trials the same TK construct from human cardiac titin (EMBL accession number X90568) was used. This construct includes residues 24731-25054 comprising the catalytic domain of the kinase and the autoregulatory C-terminal tail. The TK construct was overexpressed in baculovirus-infected insect cells and purified as previously described (Mayans et al., 1998). Crystallization attempts were limited by low yields of purified TK, typically $\sim 1 \mathrm{mg}$ TK from 601 infected SF9 cells, and by low concentration of protein in the range 5$7 \mathrm{mg} \mathrm{ml}^{-1}$. Above this concentration the TK construct precipitated.

Crystallization trials were set up as vapour-diffusion experiments using the hanging drop method (McPherson, 1982 ) on siliconized cover slips over $1 \mathrm{ml}$ reservoirs in Linbro multi-well tissue culture plates (ICN Biomedicals, Eschwege, Germany). Crystallization conditions were screened by the protocol of Jancarik \& Kim (1991) using the multifactorial crystal screens I and II from Hampton Research (Riverside, USA) and the fast-screen protocol of Zeelen et al. (1994) with an automatic pipetting system (Gilson 222 XL Liquid Handler). Drops consisted of $1.0 \mu \mathrm{l}$ of protein solution and $1.0 \mu \mathrm{l}$ of reservoir solution. Trials were maintained at either $293 \mathrm{~K}$ or $277 \mathrm{~K}$.

Initial screening yielded crystals from a number of conditions, comprising various salts and polyethylene glycols (PEG) of different molecular weight as precipitants (Table 1). From all different conditions, crystals grew with closely related needle or plate morphologies (Fig. 1) within less than three days. Most of these crystals were part of multicrystalline clusters. When salts were used as precipitants, addition of small quantities of low-molecular PEGs or ethanol increased the size of the plate planes to a maximum of $400 \mu \mathrm{m}$ in the longest direction and favoured the growth of single crystals. However, the thickness of the plates was never increased. The crystals were so thin that 
they were hardly visible under the microscope and many crystals were only discovered when stirring the drops. The relation in morphology for crystals grown from different precipitants suggested similar packing of the TK molecules irrespective of the specific crystallization conditions.

Since crystals grew most reliably from sodium/potassium tartrate as precipitant, this condition was pursued in further experiments. Single and larger crystals could only be obtained by a combined approach, including the addition of small quantities of organic solvents as additives during crystallization, macroseeding and the use of oil layers over the reservoirs (Chayen, 1997). The organic solvents employed included ethanol, PEG 1500, PEG 400 and pentaglycine (Table 1). All of these caused a similar effect on crystallization, shifting the crystal morphology from needle to plate and contributing to the growth of single crystals instead of clusters. For seeding experiments, seed fragments were obtained by either crushing large crystals or by using small ones. The concentration of the precipitant was lowered by about $20 \%$. Drops to be seeded were opened $2-4 \mathrm{~h}$ after setting and the crystal seeds, washed several times in the new mother liquor, were transferred into fresh drops using $0.2 \mathrm{~mm}$-thin glass capillaries. After seeding, oil layers were laid over the reservoirs to slow
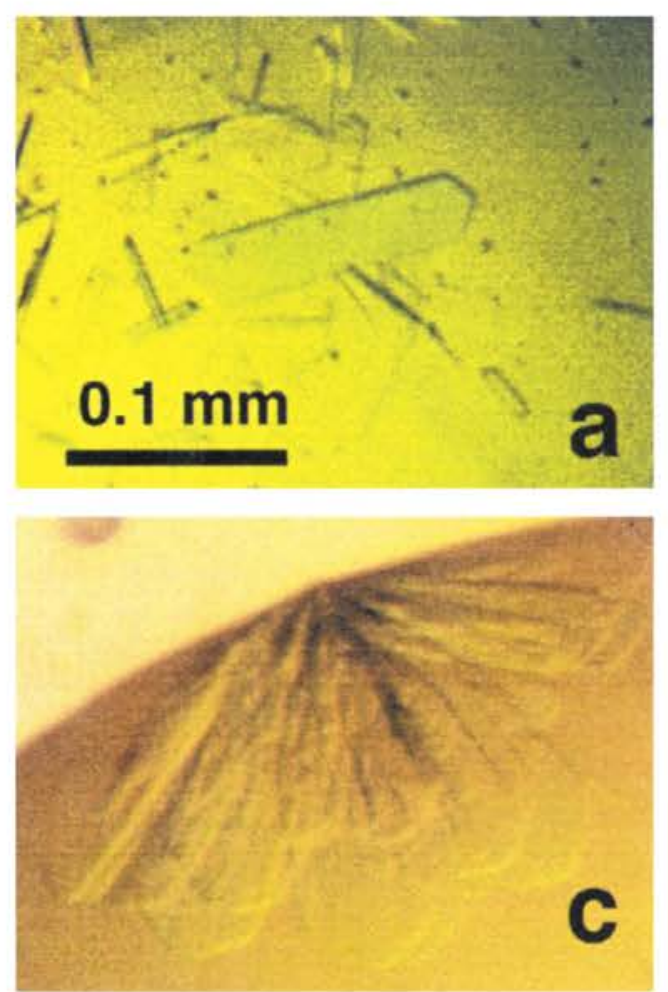

down crystal growth. They consisted of a 1:1 mixture of paraffin oil and Dow Corning 200/l cS silicone oil in a volume of $500 \mu \mathrm{l}$. The final crystals so obtained had maximal dimensions of $600 \mu \mathrm{m} \times 200 \mu \mathrm{m}$ in the plane of the plate. The thickness of these crystals still remained unmeasurable, but improved visibility of the crystals in the drops served as an indication of a moderate increase in plate thickness.

\section{Data collection and crystal characterization}

Because of their morphology, none of the TK crystals displayed significant diffraction when exposed to X-ray radiation from a rotating anode. However, early tests using synchrotron radiation at EMBL/DESY, Hamburg, revealed that the crystals had good crystallographic properties in terms of crystal mosaicity and diffraction limit. Subsequent attempts failed, however, to yield data intensities with reasonable signal-to-noise statistics, requiring further improvement of crystallization conditions (see above).

Recently we have succeeded in collecting complete data to a maximum resolution of $1.85 \AA$ from a crystal plate at the wiggler beamline BW7B at EMBL/DESY, Hamburg. The crystal was grown from $1.5 \mathrm{M}$ sodium/potassium
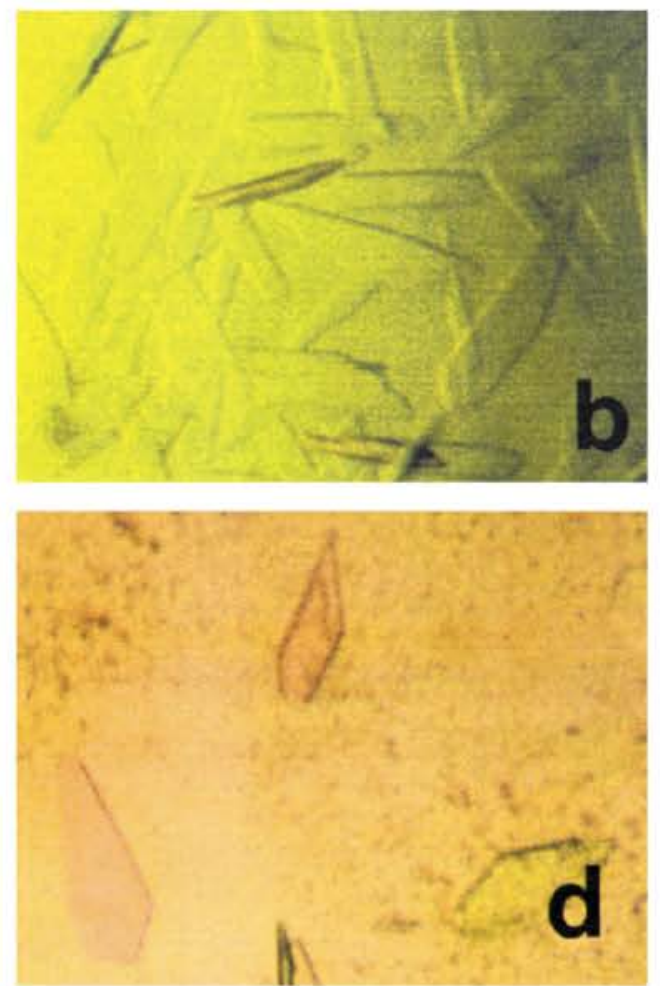

Figure 1

TK crystals: (a) macroseeded in $1.1 \mathrm{M}$ sodium/potassium tartrate, $2.5 \%(v / v)$ ethanol, $25 \mathrm{~m} M$ sodium acetate $\mathrm{pH} 4.9,25 \mathrm{~m} M$ imidazole $\mathrm{pH} 7.5 ;$ (b) $2.5 M$ sodium acetate, $25 \mathrm{~m} M$ sodium acetate $\mathrm{pH} 4.9,25 \mathrm{~m} M$ imidazole $\mathrm{pH} 7.5 ;($ c $) 1.0 M$ sodium citrate, $25 \mathrm{~m} M$ imidazole pH 7.5, $25 \mathrm{~m} M$ sodium acetate $\mathrm{pH} 4.9 ;(d) 18 \%(w / v)$ PEG-8000, $38 \mathrm{~m} M$ imidazole $\mathrm{pH} 7.5,12 \mathrm{~m} M$ sodium acetate $\mathrm{pH} 4.9,0.2 M$ magnesium chloride. Further details on crystallization conditions are given in Table 1. Multicrystalline clusters such as those in $(c)$ have also been observed from other crystallization conditions. The dimensions of the crystals are indicated by the bar. 
Table 1

Crystallization conditions.

\begin{tabular}{|c|c|c|c|}
\hline \multicolumn{2}{|c|}{ Precipitant } & \multirow{2}{*}{$\begin{array}{l}\text { Buffer } \\
B, C, F\end{array}$} & \multirow{2}{*}{$\frac{\text { Maximum crystal size }}{500 \times 200 \times<5 \mu^{3}}$} \\
\hline 1 & $\begin{array}{l}\text { 1.4-1.6 } M \text { sodium/potassium } \\
\text { tartrate }\end{array}$ & & \\
\hline & $2.3-3.2 \mathrm{M}$ sodium formiate & $C, E, F$ & $200 \times 20 \times<5 \mu \mathrm{m}^{3}$ \\
\hline 3 & $1.5 \mathrm{M}$ & & 100 \\
\hline 4 & $1.0-1.2 \mathrm{M}$ sodium citrate & $A, B, E, F$ & $300 \times 100 \times<5 \mu \mathrm{m}^{3}$ \\
\hline 5 & $2.5-3.3 \mathrm{M}$ sodium acetate & $C, E, F$ & $300 \times 50 \times<5 \mu \mathrm{m}^{3}$ \\
\hline 6 & $20 \%(v / v)$ PEG-8000市 & c & $300 \times 50 \times<5 \mu \mathrm{m}^{3}$ \\
\hline 7 & $17-20 \%(v / v)$ PEG- $4000 \%$ & C & $300 \times 50 \times<5 \mu \mathrm{m}^{3}$ \\
\hline
\end{tabular}

Buffer compositions§

A $\quad 50 \mathrm{~m} M$ sodium acetate, $\mathrm{pH} 4.9$ (acetic acid)

B $25 \mathrm{~m} M$ sodium acetate, $\mathrm{pH} 4.9$ (acetic acid) $25 \mathrm{~m} M$ imidazole, $\mathrm{pH} 7.5(\mathrm{NaOH})$

C $25 \mathrm{~m} M$ sodium acetate, $\mathrm{pH} 4.9$ (acetic acid); $25 \mathrm{~m} M$ HEPES, $\mathrm{pH} 7.5(\mathrm{NaOH})$

D $\quad 50 \mathrm{mM}$ sodium acetate, $\mathrm{pH} 6.5$ (acetic acid)

E $\quad 50 \mathrm{mM}$ HEPES, $\mathrm{pH} 7.5(\mathrm{NaOH})$

$F \quad 25 \mathrm{~m} M$ HEPES, $\mathrm{pH} 7.5 ; 25 \mathrm{~m} M$ bis-tris propane, $\mathrm{pH} 8.0$

† Various organic solvents were used as additives: $5 \%(v / v)$ ethanol, $2.5 \%(v / v)$ PEG$1500,2.5 \%(v / v)$ PEG-400, $1 \mathrm{~m} M$ pentaglycine. $\$$ Additive; $200 \mathrm{~m} M$ magnesium chloride. \$ Each buffer includes $1 \mathrm{~m} M$ edta, $1 \mathrm{~m} M$ sodium azide, $1 \mathrm{~m} M$ DTT.

tartrate, $2.5 \%(v / v)$ PEG-1500, $25 \mathrm{~m} M$ sodium acetate at $\mathrm{pH} 4.9$ and $25 \mathrm{mM}$ imidazole at $\mathrm{pH} 7.5$ and had approximate dimensions of $300 \mu \mathrm{m} \times 100 \mu \mathrm{m}$ in the plane of the plate ( $c f$. Table 1). The crystal was flash frozen at $100 \mathrm{~K}$ using $12 \%$ glycerol as cryoprotectant and mounted in a nylon CryoLoop (Hampton Research, Riverside, USA). An extra-large loop with a diameter of $\sim 1 \mathrm{~mm}$ was used to avoid any mechanical stress. Many test crystals, mounted into smaller cryoloops, had shown various degrees of disorder when exposed along the plane formed by the loop. The crystal was mounted with the plane of the plate within the layer of frozen mother liquor of the cryoloop and the longest dimension of the crystal approximately aligned with the pin holder of the loop which parallels the spindle axis. Since the crystal was hardly visible in the video system of this beamline, test images of different areas of the loop were collected to ensure an optimal centring of the crystal. To optimize the signal-to-noise ratio of the diffraction data, the beam cross section was set to $0.3 \mathrm{~mm}^{2}$ so that the longest dimension of the crystal plate was approximately matched by the beam. Frames were collected on a constantdose mode in arbitrary units, equivalent to exposure times of $\sim 1-2$ min depending on the ring current. Further details of the data collection and the resulting statistics have been described previously (Mayans et al., 1998).

Fig. 2 shows $I / \sigma$ ratios and $R$-merge values of this data set in resolution bins and as a function of time, indicating the high quality of the crystal used for data collection. The average scale factors of each frame showed little variation, confirming that the crystal was well centred during the entire data collection despite its poor visibility in the video system (Fig. 2c). The average temperature factors for the individual frames, however, steadily increased indicating that the crystal suffered considerably from radiation damage during data acquisition (Fig. $2 c$ ). Based on these statistics, only diffraction data below $2.0 \AA$ resolution were

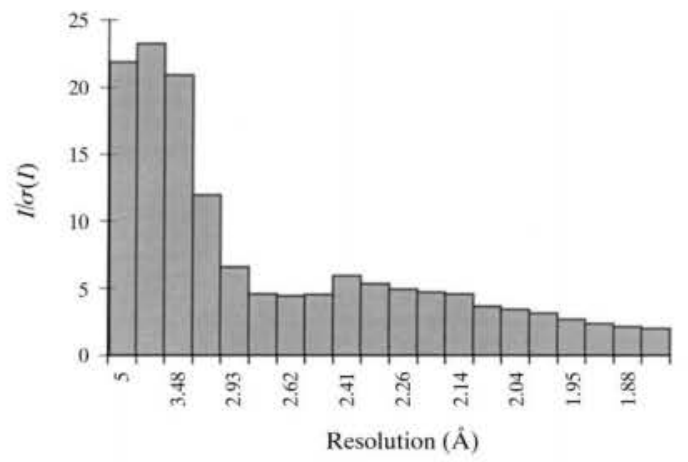

(a)

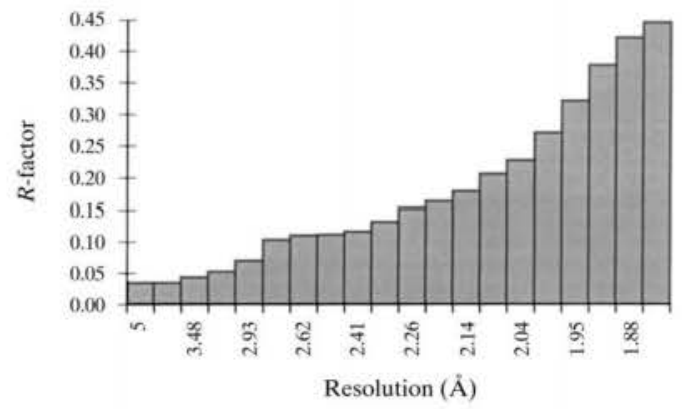

(b)

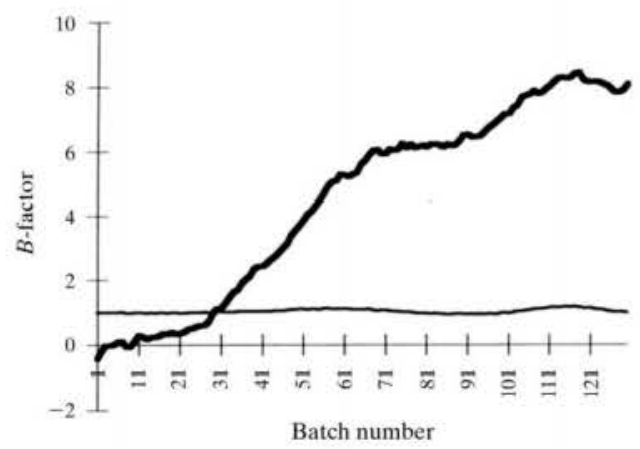

(c)

Figure 2

$\mathrm{X}$-ray data statistics as a function of resolution and data-collection time. The plots indicate that the quality of the data set has been limited by the small crystal volume exposed to X-ray radiation, reflected by moderate $I / \sigma$ statistics. $I / \sigma$ values and $R$-merge as output by the program SCALEPACK (Otkinowski, 1993). (a) $I / \sigma$ and (b) $R$-merge in resolution bins; $(c)$ scale factor (thin line) and temperature factor (in bold, dimension in $\AA^{2}$ ) as a function of frame number. The data set consists of 68110 unique reflections in the range $20.0-1.85 \AA$ resolution with a redundancy of 8.9 and overall completeness of $98.2 \%$. For further details on the data collection, see Mayans et al. (1998). 


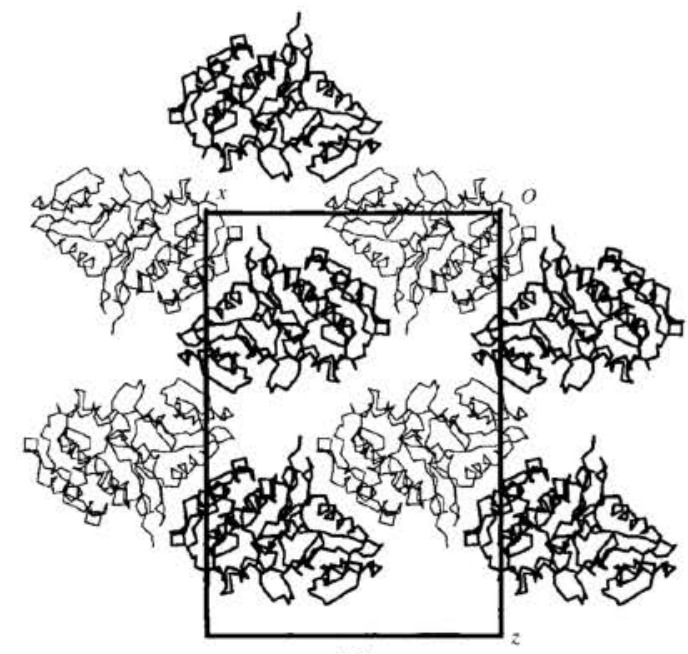

(a)

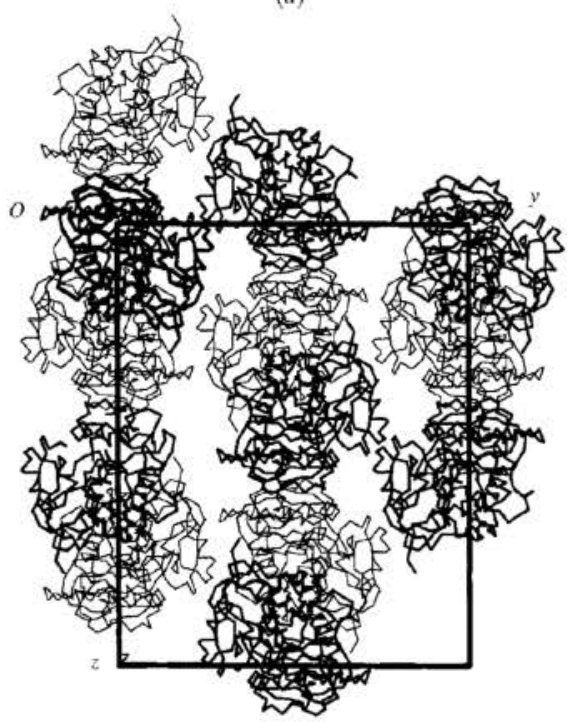

(b)

\section{Figure 3}

Molecular packing of the NCS dimers in the TK crystals $(a)$ in the $a c$ plane and $(b)$ in the $a b$ plane. For each NCS dimer, the $C_{\alpha}$ skeleton of one molecule is drawn in bold and that of the second molecule is drawn in thin lines. The TK NCS dimers form tightly packed layers within the $a c$ plane. A second layer of dimers is generated by the $2_{1}$ component along the $b$ axis. Within one layer of TK molecules in the $a c$ plane there are three major contact areas: (i) between the same $\beta$-strand $\beta \mathrm{Cl}$ of the $\mathrm{N}$-terminal lobe of two adjacent molecules, forming an antiparallel $\beta$-sheet-like arrangement, (ii) two helix/helix packing zones, involving always the packing of helix $\alpha C 1$ from one molecule and helix $\alpha C 7$ from a neighbouring molecule. In contrast, the sparse contacts perpendicular to these molecular layers are exclusively formed by residues in loop regions. The number of strong specific packing interactions (hydrogen bonds, salt bridges) between adjacent molecules has been estimated using CONTACT (Collaborative Computational Project, Number 4, 1994). There are 18 specific contacts per TK molecule within the ac layer, but only three specific interlayer contacts. The ratio of the contacts in different orientations demonstrates the dominance of packing interactions in the $a c$ plane. used for the structure determination of TK (Mayans et al., 1998).

\section{Analysis of the crystal packing}

To find any rationales for the inhibited growth of the TK crystals perpendicular to the plate plane we correlated the crystal morphology with the crystallographic symmetry and analysed the molecular packing (Fig. 3). Crystals belong to the space group $P 2_{1} 2_{1} 2_{1}$ with cell dimensions of $a=78.6 \AA$, $b=89.8 \AA, c=113.3 \AA$ and two TK molecules in the asymmetric unit. The non-crystallographic twofold axis is parallel to the $b$ axis (Mayans et al., 1998).

The relation between the crystal morphology and the crystallographic axes was derived from the misseting angles in the initial crystal orientation. The crystallographic $a$ and $c$ axes are within the crystal plate plane with $a$, the shortest unit-cell dimension, parallel to the longest dimension of the plates ( $c f$. Fig. 1). The $b$ axis is perpendicular to the crystal plane.

Packing within the $a c$ plane is tight (Fig. 3a), with numerous interactions between the catalytic domains including both helix-helix packing and contacts between $\beta$ strands from the $\mathrm{N}$-terminal lobe. In contrast, only a very limited number of interactions are present between these molecular layers along the $b$ axis (Fig. $3 b$ ). Since the twofold NCS operator is along the $b$ axis, the packing of the $a c$ layers is formed by NCS dimers. The number of specific interactions (hydrogen bonds, salt bridges) found within the layer of molecules in the ac plane is about sixfold higher than perpendicular to the plane (for details, see legend of Fig. 3). Based on the size of the unit cell along the $b$ axis and the thickness of the crystals, the number of these molecular layers is limited to about 1000 . In conclusion, in this example crystal growth appears to be correlated with the population of intermolecular contacts.

Despite the imbalance in growth, the TK crystal used for data collection displayed only moderate anisotropy in diffraction. To analyse this further, an overall anisotropic tensor was calculated for the observed structure-factor moduli $\left|F_{o}\right|$ by comparison with $\left|F_{c}\right|$ values derived from the isotropic model (Sheriff \& Hendrickson, 1987). Calculations used $X$-PLOR (Brünger, 1990) and yielded values of $B_{11}=6.23, B_{22}=-6.21, B_{33}=-0.02$, improving the $R$-factor fitting by $\sim 1.2 \%$. This indicates that the diffraction along the $b$ axis is weaker than that along the other two axes. Apparent strong anisotropies or disorder observed in previous test crystals can, in retrospect, be attributed to mechanical stress caused by mounting, presumably due to contacts between crystal and loop, and shock freezing.

Despite various reports on data collection from small and fragile crystals (for review, see Helliwell, 1992), only a few studies of the crystal packing as the potential source of the difficulties have been carried out. Crystals of bacteriorhodopsin were grown as thin plates of thickness $\sim 5 \mu \mathrm{m}$ and diffracted isotropically up to $2.0 \AA$ resolution when exposed to highly focused synchrotron radiation (Landau 
\& Rosenbusch, 1996). Like in TK, the crystal packing of bacteriorhodopsin consists of tightly packed protein layers stacked along the thinnest dimension of the crystal with only a few contacts observed between the layers (PebayPeyroula et al., 1997). Crystals of the 50 S ribosome subunit also grow as extremely thin plates (Yonath et al., 1998; Ban et al., 1998). A preliminary electron density map at $8 \AA$ resolution indicates that there are rather few packing interactions along the axis perpendicular to the plane of the plate, outscored by the packing contacts within the plane (Yonath et al., 1998). The sample scattering efficiency of TK, $\Sigma f_{j}^{2} V(x) / V_{0}^{2}$, is $0.06 \times 10^{12} \mathrm{e}^{2} \AA^{-3}$, which is almost as weak as the FMDV + minimum scattering efficiency example tabulated by Helliwell (1992) of $0.02 \times$ $10^{12} \mathrm{e}^{2} \AA^{-3}$.

\section{Conclusions}

The availability of highly intense and focused synchrotron radiation permits data collection from difficult crystal forms that would otherwise not be suitable for X-ray structure solution. This has been demonstrated for extremely thin plate crystals of TK by exploiting and optimizing the properties of a wiggler beamline at the EMBL Hamburg Outstation. In TK, as well as in bacteriorhodopsin, crystal growth perpendicular to the plate plane appears to be outscored by the numerous molecular contacts within the plane which leads to quasi two-dimensional crystal growth. A higher number of other macromolecular crystals with thin-plate morphology still remains to be characterized to further generalize our observations on unbalanced crystal growth.

$\div$ For TK, 5738 atoms per unit cell $\times 7^{2}$ (i.e. assumed to be $\mathrm{N}$ atoms) $\times$ $1.35 \times 10^{17}$ (sample volume in $\left.\AA^{2}\right) /\left(7.997 \times 10^{5}\right)^{2}=0.06 \times 10^{12} \mathrm{e}^{2} \AA^{-3}$. \% FMDV $=$ foot-and-mouth disease virus.
We thank Michel Roth for beam-time allocation and assistance at the IBS/ESRF beamline D2AM (Grenoble, France) and Kristina Djinovic Carugo for joining efforts during data collection. Thanks to Victor Lamzin and Rob Meijers for critical discussions.

\section{References}

Ban, N., Freeborn, B., Nissen, P., Penczek, P., Grassucci, R. A. Sweet, R., Frank, J., Moore, P. B. \& Steitz, T. A. (1998). Cell, 93, 1105-1115.

Brünger, A. T. (1990), X-PLOR. A System for X-ray Crystallography and NMR. Version 3.1. Yale University, New Haven, CT, USA.

Chayen, N. E. (1997). J. Appl. Cryst. 30, 198-202.

Collaborative Computational Project, Number 4 (1994). Acta Cryst. D50, 760-763.

Helliwell, J. R. (1992). Macromolecular Crystallography with Synchrotron Radiation, pp. 410-414. Cambridge University Press.

Jancarik, J. \& Kim, S. H. (1991). J. Appl. Cryst. 24, 409-411.

Landau, E. M. \& Rosenbuch, J. P. (1996). Proc. Natl Acad. Sci. 93, $14532-14535$.

McPherson, A. (1982). Preparation and Analysis of Protein Crystals. New York: Wiley and Sons.

Mayans, O., van der Ven, P. E. M., Wilm, M., Mues, A., Young, P., Fürst, D. O., Wilmanns, M. \& Gautel, M. (1998). Nature (London), 395, 863-869.

Otkinowski, Z. (1993). Proceedings of the CCP4 Study Weekend: Data Collection and Processing, pp. 56-62. Warrington: Daresbury Laboratory.

Pebay-Peyroula, E., Rummel, G., Rosenbusch, J. P. \& Landau, E. M. (1997). Science, 277, 1676-1681.

Sheriff, S. \& Hendrickson, W. A. (1987). Acta Cryst. A43, 118-121. Yonath, A., Harms, J., Hansen, H. A. S., Bashan, A., Schluenzen, F., Levin, I., Koelln, I., Tocilj, A., Agmon, I., Peretz, H., Bennett, W. S., Janell, D., Weinstein, S., Auerbach, T., Avila, H., Pioletti, M., Morlang, S., Bhanumoorthy, P. \& Fraceschi, F. (1998). Acta Cryst. A54, 945-955.

Zeelen, J. P., Hiltunen, J. K., Ceska, T. A. \& Wierenga, R. K. (1994). Acta Cryst. D50, 443-447. 